Review

\title{
Potential Factors Influencing the Effects of Anthocyanins on Blood Pressure Regulation in Humans: A Review
}

\author{
Stefano Vendrame and Dorothy Klimis-Zacas * \\ School of Food and Agriculture, University of Maine, Orono, ME 04469, USA; \\ stefano.vendrame@fulbrightmail.org \\ * Correspondence: dorothea@maine.edu
}

Received: 30 May 2019; Accepted: 21 June 2019; Published: 25 June 2019

check for updates

\begin{abstract}
Dietary intake of anthocyanins (ACNs) is associated with a reduced risk of cardiovascular and coronary heart disease. While the anti-inflammatory, antioxidant, and lipid-lowering effects of ACN consumption have been consistently reported, their effect(s) on blood pressure regulation is less consistent and results from human studies are mixed. The objective of this review is attempting to identify potential patterns which may explain the variability in results related to blood pressure. To do so, we review 66 human intervention trials testing the effects on blood pressure of purified $\mathrm{ACN}$ or ACN-rich extracts, or whole berries, berry juices, powders, purees and whole phenolic extracts, from berries that are rich in ACN and have ACNs as predominant bioactives. Several factors appear to be involved on the mixed results reported. In particular, the baseline characteristics of the population in terms of blood pressure and total flavonoid intake, the dose and duration of the intervention, the differential effects of individual $\mathrm{ACN}$ and their synergistic effects with other phytochemicals, the ACN content and bioavailability from the food matrix, and individual differences in $\mathrm{ACN}$ absorption and metabolism related to genotype and microbiota enterotypes.
\end{abstract}

Keywords: anthocyanins; hypertension; blood pressure; berries

\section{Introduction}

Hypertension is a known risk factor of cardiovascular disease (CVD), which is the leading cause of death worldwide [1]. The role of lifestyle modification, including diet, as a means to reduce CVD risk is attracting increasing interest, but requires a careful evidence-based approach [2]. This is especially true for management and control of hypertension, which burdens most European and Western countries [3],

Anthocyanins $(\mathrm{ACN})$ are a large class of water-soluble plant metabolites belonging to the flavonoid family of phenolics, responsible for the red, blue, and purple pigmentation of many flowers, fruits, and vegetables. They are the glycosylated forms of highly reactive and relatively unstable molecules characterized by a flavylium cation structure, called anthocyanidins [4]. Although more than 600 naturally occurring ACNs have been identified, for the vast majority, they are made by different glycosylated forms of only six anthocyanidins: pelargonidin, cyanidin, delphinidin, peonidin, petunidin, and malvidin [5]. The average daily intake of ACNs is $200 \mathrm{mg} / \mathrm{day}$, which is about one fifth of the average daily intake of total phenolics [6]. While plants produce ACN as a defense against environmental stressors, such as temperature extremes, UV light, and drought, dietary intake of anthocyanins has been extensively studied for its health-promoting potential, in particular with respect to cardiovascular disease prevention [7].

Strong epidemiological evidence associates ACN intake with a reduced risk for cardiovascular disease and coronary heart disease [8]. While ACN consumption has been consistently associated with 
anti-inflammatory, antioxidant, and lipid-lowering effects, its effect on blood pressure regulation is less consistent and results from human studies are mixed $[9,10]$.

To try to identify potential patterns explaining such variability in results related to blood pressure, we decided to review clinical trials testing purified ACN or ACN-rich extracts, or whole berries, berry juices, powders, purees, or whole phenolic extracts from berries that are rich in ACNs and have ACNs as predominant bioactives. Among berries, a higher ACN content can be found in chokeberries (Aronia melanocarpa) (over $400 \mathrm{mg} / 100 \mathrm{~g}$ ) [11], blackcurrants, blueberries, and raspberries (over $100 \mathrm{mg} / 100 \mathrm{~g}$ ), followed by blackberries and cherries (over $50 \mathrm{mg} / 100 \mathrm{~g}$ ) [12,13]. Instead, cranberries, grapes, and strawberries contain, on average, less than $50 \mathrm{mg} / 100 \mathrm{~g}$, and more of other bioactive phytochemicals [6,14]. Thus we chose not to consider them in this review as a proxy for $\mathrm{ACNs}$, unless they were used as part of berry mixes or as a source of ACN extracts.

\section{ACNs and Blood Pressure}

High blood pressure can be caused by an increased cardiac output or by a higher systemic vascular resistance, which in turn results either from a narrower diameter of blood vessels or by their lower elasticity. An excess of vasoconstricting stimuli, or a lack of vasodilator stimuli, can both result in hypertension [1]. ACNs have been associated with blood pressure regulation, directly or indirectly, by three major mechanisms:

1. ACNs have been consistently shown to increase endothelial-derived nitric oxide (NO), via modulation of endothelial NO synthase (eNOS) expression and activity. Nitric oxide is one of the major contributors to endothelium-dependent vasorelaxation. It causes vascular smooth muscle relaxation following activation of soluble guanylate cyclase, which in turn increases cGMP. This blocks the release of intracellular calcium, preventing it from causing vascular smooth muscle contraction [15].

2. Reactive oxygen species damage NO, thus promoting vasoconstriction and hypertension. Due to their strong antioxidant activity, ACNs act to prevent $\mathrm{NO}$ oxidative damage and radical-induced NO conversion, such as the reaction caused by NADPH oxidase [16].

3. ACNs have been shown to reduce synthesis of vasoconstricting molecules, such as angiotensin II via inhibition of the angiotensin-converting enzyme (ACE) activity, endothelin-1, and thromboxanes via inhibition of the cyclooxygenase (COX) pathway [17].

While most of these mechanistic observations come from in vitro and animal model studies [18], the practical outcome of ACN dietary consumption on blood pressure regulation in humans is likely complicated by multiple factors.

\subsection{Epidemiological and Meta-Analysis Data}

Epidemiological data appears to confirm the existence of a link between ACNs and blood pressure regulation. A prospective epidemiological study on 34,489 postmenopausal women from the Iowa Women's Health Study, analyzing the effects of total flavonoids or seven different individual flavonoid subclasses on cardiovascular health over 12 years, found a significant inverse association between ACNs and CHD and CVD and total mortality, while total flavonoids and the other individual subclasses had no significant effect [6]. Analyzing data from a cohort of 156,957 men and women from the Nurses' Health Study, the NHS I and the Health Professionals Follow-Up Study, followed for 14 years, Cassidy et al. found an inverse association between ACN intake and hypertension [19]. Interestingly, this association was not observed for total flavonoid intake, nor for any other subclass of flavonoids (flavones, flavonols, flavan-3-ols, and flavanones), with the exception of two single compounds (apigenin and catechin) [19]. In a cross-sectional study from a cohort of 1898 adult women from the TwinsUK registry, a higher intake of ACNs was associated with significantly lower central systolic blood pressure (SBP) and mean arterial pressure (MAP). Again, the inverse association was not observed for total flavonoid intake, flavanones, flavan-3-ols, flavonols, or flavones [20]. 
Several meta-analyses have been conducted using data from clinical trials involving sources of ACNs, with mixed results. A meta-analysis of 128 clinical trials on different sources of ACN and ellagitannins, with a total of 5538 participants, found that both systolic and diastolic blood pressure (DBP) were significantly lowered by consumption of berries, red grapes, and red wine- the main sources of ACN investigated in the study [12]. The effect is also significant when studies on berries only and studies on red wine/red grapes only are considered [12]. A meta-analysis of 22 clinical trials studying the effects of berries (total 1251 subjects), found a significant reduction of SBP, but not DPB [21]. Per contra, a meta-analysis on 32 clinical trials (1491 total participants) investigating the effects of ACNs and ACN sources on cardiometabolic health, found that the reduction in SBP and DBP did not reach statistical significance [22]. Similarly, a meta-analysis of 19 clinical studies found no significant effect of ACN supplementation on either SB or DBP [23]. A meta-analysis of six clinical studies with 472 total participants, found no significant effect of ACN supplementation on either SBP or DBP [24]. A meta-analysis of six clinical trials with 204 total participants, detected no significant effect of blueberry consumption on blood pressure [25]. The participants of all the above mentioned meta-analyses represented a mixed population, including men and women of all age groups and from different geographical regions, both healthy and with cardiovascular risk factors.

In light of such mixed results, we searched the literature for clinical trials, testing the effects on blood pressure of ACNs or ACN-rich berries and examined the results individually to attempt to identify potential patterns explaining the variability in results related to blood pressure.

\subsection{Literature Search}

A search of the literature for human acute and chronic intervention studies was carried out on multiple databases (PubMed, ScienceDirect, and Web of Science) with the keywords (anthocyanin* OR blueberr* OR raspberr* OR bilberr* OR blackberr* OR blackcurrant OR açai OR cherr* OR aronia OR elderberr* OR chokeberr*) AND (blood pressure OR systolic OR diastolic OR MAP OR aldosterone OR angiotensin* OR renin OR nitric oxide OR blood flow). Abstracts and full texts were screened, and reference lists were also searched for related articles. Further details about the literature search process are provided in Figure 1. Sixty-six relevant studies were identified [26-88], and their results related to blood pressure are summarized in Tables 1 and 2.

\subsection{Single-Dose Interventions}

Of the 14 single-dose interventions which were identified and reviewed; six found a significant effect on blood pressure, as summarized in Table 1.

All single-dose studies were performed on healthy participants, with the exception of the study by Keane et al. which was conducted on 15 subjects with early hypertension: both SBP and mean arterial pressure, but not DBP, were significantly lower at 1, 2, 3, 4, 5, 6, 7, and 8 hours after a serving of tart cherry juice providing $73.5 \mathrm{mg}$ ACN [31]. Del Bo et al. investigated the effect of a serving of blueberry juice providing 300-350 $\mathrm{mg} \mathrm{ACN}$ in restoring blood pressure after a cigarette was smoked by young otherwise healthy smokers. In one study, the SBP spike induced by smoke was restored by the blueberry juice [28], but the effect was not replicated in a following study [29]. 
Search keywords: (anthocyanin* OR blueberr* OR raspberr* OR bilberr* OR blackberr* OR blackcurrant OR açai OR cherr* OR aronia OR elderberr* OR chokeberr*) AND (blood pressure OR systolic OR diastolic OR MAP OR aldosterone OR angiotensin* OR renin OR nitric oxide OR blood flow)

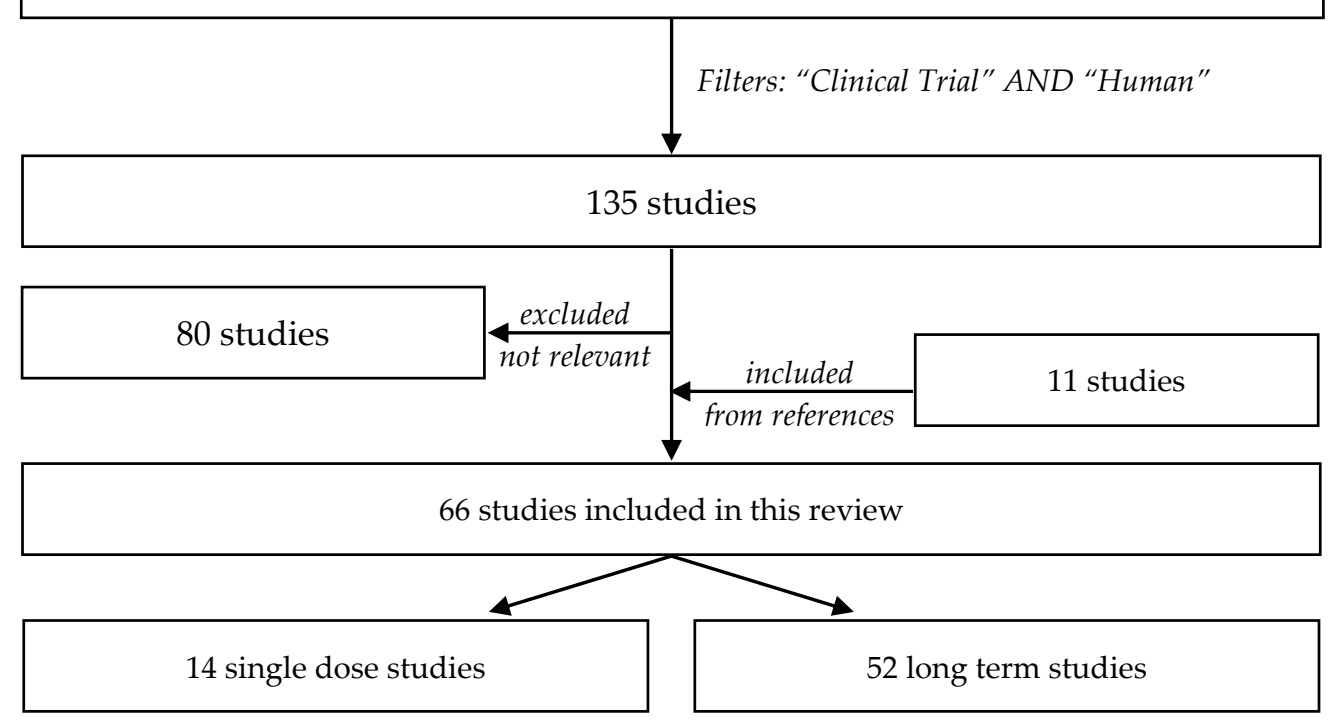

Figure 1. Flow chart describing the literature search process. The literature search was conducted on PubMed, ScienceDirect, and Web of Science databases and limited to original clinical trials with human participants published after January 1st, 2005. One-hundred-and-thrity-five abstracts and full texts were reviewed. Eighty studies not reporting both ACN source and blood pressure markers were excluded. Eleven additional relevant studies were identified during the screening process from the reference lists, and were added to the review.

\subsection{Long-Term Interventions}

Fifty-two long term interventions were identified and summarized in Table 2. For obvious practical reasons, and for the purpose of standardization, only few studies used fresh berries, while most studies used more stable, but processed berries such as juices, concentrates, or freeze-dried powders. Eleven studies used only phenolic extracts, and 14 studies used isolated ACNs or ACN-rich extracts. Of the 52 reviewed interventions, 21 found a significant effect on blood pressure (Table 2). 
Table 1. Summary of single dose interventions.

\begin{tabular}{|c|c|c|c|c|c|c|}
\hline Reference & Study Design & Control & Participants $^{a}$ & Source of ACN & Daily Dose of $\mathrm{ACN}^{\mathrm{b}}$ & Effect on Blood Pressure ${ }^{c}$ \\
\hline Alquarashi [26] & Crossover & Double-blind, controlled & $\begin{array}{c}23 \text { healthy male, age } \\
46 \pm 9, \text { BMI } 28 \pm 2\end{array}$ & $\begin{array}{l}\text { Açai-based smoothie, (control: } \\
\text { macronutrient-matched } \\
\text { smoothie), following } \\
\text { high-fat meal }\end{array}$ & $493 \mathrm{mg}$ & $=\mathrm{SBP},=\mathrm{DBP}$ at 2 and $6 \mathrm{~h}$ \\
\hline Castro-Acosta [27] & Crossover & Double-blind & $\begin{array}{c}23 \text { healthy, age } 46 \pm 14, \\
\text { BMI } 26 \pm 3.8\end{array}$ & $\begin{array}{l}\text { Blackcurrant extract drink, } \\
\text { following } \\
\text { high-carbohydrate meal }\end{array}$ & 150,300 , or $600 \mathrm{mg}$ ACN & $=\mathrm{SBP},=\mathrm{DBP}$ after $2 \mathrm{~h}$ \\
\hline Del Bo [28] & Crossover & Controlled & $\begin{array}{c}10 \text { younger adults, age } \\
21 \pm 2 \text {, BMI } 23 \pm 2\end{array}$ & $\begin{array}{l}\text { Blueberry smoothie (control: } \\
\text { sugar matched jelly) }\end{array}$ & $348 \mathrm{mg}$ & $=\mathrm{SBP},=\mathrm{DBP}$ \\
\hline Del Bo [28] & Crossover & Controlled & $\begin{array}{c}16 \text { smokers, age } 24 \pm 1 \\
\text { BMI } 23 \pm 1\end{array}$ & $\begin{array}{l}\text { Blueberry drink (control: sugar } \\
\text { matched drink), followed by } \\
\text { smoking } 1 \text { cigarette }\end{array}$ & $348 \mathrm{mg}$ & $<\mathrm{SBP}$ post smoke, $=\mathrm{DBP}$ \\
\hline Del Bo [29] & Crossover & Controlled & $\begin{array}{c}12 \text { healthy male, age } 24 \\
\pm 1, \text { BMI } 23 \pm 1\end{array}$ & $\begin{array}{l}\text { Blueberry drink (control: sugar } \\
\text { matched drink) }\end{array}$ & $309 \mathrm{mg}$ & $=\mathrm{SBP},=\mathrm{DBP}$ \\
\hline Del Bo [29] & Crossover & Controlled & $\begin{array}{l}12 \text { healthy male smokers, } \\
\text { age } 15 \pm 2, \text { BMI } 23 \pm 1\end{array}$ & $\begin{array}{l}\text { Blueberry drink (control: sugar } \\
\text { matched drink), followed by } \\
\text { smoking } 1 \text { cigarette }\end{array}$ & $309 \mathrm{mg}$ & $\begin{array}{l}\text { Did not restore blood } \\
\text { pressure }\end{array}$ \\
\hline Igwe [30] & Crossover & Controlled & $\begin{array}{l}12 \text { young (age } 31 \pm 8,23 \\
\pm 2 \text { ) and } 12 \text { older adults } \\
\text { (age } 77 \pm 6, \text { BMI, } 26 \pm 3 \text { ) }\end{array}$ & Plum juice & $369 \mathrm{mg}$ & $\begin{array}{c}<\mathrm{SBP},<\mathrm{DBP},<\mathrm{MAP} \text { in both } \\
\text { age groups, compared to } \\
\text { baseline }\end{array}$ \\
\hline Keane [31] & Crossover & $\begin{array}{l}\text { Single-blind, } \\
\text { placebo-controlled }\end{array}$ & $\begin{array}{c}15 \text { male with early } \\
\text { hypertension, age } 31 \pm 9, \\
\text { BMI } 27 \pm 4\end{array}$ & Tart cherry juice & $73.5 \mathrm{mg}$ & $<\mathrm{SBP},<\mathrm{MAP},=\mathrm{DBP}$ \\
\hline Keane [32] & Crossover & $\begin{array}{l}\text { Double-blind, } \\
\text { placebo-controlled }\end{array}$ & $\begin{array}{l}27 \text { healthy, age } 50 \pm 6 \\
\text { BMI } 26 \pm 5\end{array}$ & Tart cherry juice & $68 \mathrm{mg}$ & $<\mathrm{SBP}$ \\
\hline Keane [33] & Crossover & $\begin{array}{l}\text { Double-blind, } \\
\text { placebo-controlled }\end{array}$ & $\begin{array}{c}10 \text { athletes, age } 28 \pm 7 \\
\text { BW } 78 \pm 9\end{array}$ & Tart cherry juice & $68 \mathrm{mg}$ & $<\mathrm{SBP},=\mathrm{DBP},=\mathrm{MAP}$ \\
\hline Kent [34] & Crossover & Controlled & $\begin{array}{c}6 \text { young (age } 22 \pm 1 \text {, BMI } \\
26 \pm 4 \text { ) and } 7 \text { older } \\
\text { adults (age } 78 \pm 6 \text {, BMI } \\
29 \pm 4 \text { ) }\end{array}$ & $\begin{array}{l}\text { Cherry juice, in single dose or } \\
\text { three doses over } 2 \text { hours }\end{array}$ & $207 \mathrm{mg}$ & $\begin{array}{c}<\mathrm{SBP},<\mathrm{DBP} \text { at } 2 \mathrm{~h} \text { after } \\
\text { consumption, if given in a } \\
\text { single dose (but not if split in } \\
\text { three doses given } 1 \text { hour } \\
\text { apart) }\end{array}$ \\
\hline Matsumoto [35] & Crossover & $\begin{array}{l}\text { Double-blind, } \\
\text { placebo-controlled }\end{array}$ & $\begin{array}{l}9 \text { healthy male, age } \\
30 \pm 1 \text {, BMI? }\end{array}$ & Blackcurrant ACN extract & $(17$ mg/kg) & $=\mathrm{SBP},=\mathrm{DBP}$ \\
\hline Rodriguez-Mateos [36] & Crossover & Double-blind, controlled & $\begin{array}{l}10 \text { healthy male, age } \\
27 \pm 3, \text { BMI } 25 \pm 3\end{array}$ & $\begin{array}{c}\text { Blueberry drink or } \\
\text { nutrient-matched control } \\
\text { Freeze-dried blueberry drink }\end{array}$ & $310 \mathrm{mg}$ & $=\mathrm{SBP},=\mathrm{DBP}$ \\
\hline Rodriguez-Mateos [37] & Crossover & Double-blind, controlled & $\begin{array}{l}10 \text { healthy, age } 27 \pm 1 \text {, } \\
\text { BMI } 25 \pm 1\end{array}$ & $\begin{array}{l}\text { blueberry baked product (with } \\
\text { same amount of blueberry } \\
\text { powder), or baked control }\end{array}$ & $\begin{array}{l}339 \mathrm{mg} \text { in drink, } 196 \mathrm{mg} \\
\text { in baked product }\end{array}$ & $=\mathrm{SBP},=\mathrm{DBP}$ \\
\hline
\end{tabular}

a Age (in years) and BMI (in $\mathrm{kg} / \mathrm{m}^{2}$ ) data expressed as mean $\pm \mathrm{SD}$. When data was reported as SEM, SD was calculated as SEM ${ }^{*} \mathrm{SQRTparticipant.} \mathrm{When} \mathrm{BMI} \mathrm{was} \mathrm{unreported,} \mathrm{weight} \mathrm{in} \mathrm{kg}$ is reported instead. A question mark (?) indicates unreported data. ${ }^{\mathrm{b}}$ When daily ACN dose is unreported, the best alternative information is reported in parentheses. ${ }^{\mathrm{c}}$ Only blood pressure data is reported in this table. Other outcomes of the studies are not reported. SBP, systolic blood pressure; DPB, diastolic blood pressure; MAP, mean arterial pressure; > or <, statistically significant increase or decrease; $=$, no significant change. 
Table 2. Summary of long-term interventions.

\begin{tabular}{|c|c|c|c|c|c|c|c|}
\hline Reference & Duration & Study Design & Control & Participants $^{a}$ & Source of ACN & $\begin{array}{l}\text { Daily Dose } \\
\text { of ACN }{ }^{b}\end{array}$ & Effect on Blood Pressure $^{c}$ \\
\hline Ataie-Jafari [38] & 6 weeks & Pre-post & - & $\begin{array}{c}19 \text { women with diabetes, age } \\
53 \pm 9 \text { BMI } 30 \pm 4\end{array}$ & Sour cherry juice & $720 \mathrm{mg}$ & $<\mathrm{SBP},<\mathrm{DBP}$ \\
\hline Barona [39] & 4 weeks & Crossover & $\begin{array}{c}\text { Double-blind, } \\
\text { placebo-controlled }\end{array}$ & $\begin{array}{c}24 \text { male with, age } 51 \pm 10 \\
\text { BMI } 32 \pm 5\end{array}$ & $\begin{array}{c}\text { Freeze-dried grape } \\
\text { phenolic extract }\end{array}$ & $35 \mathrm{mg}$ & $<\mathrm{SBP},=\mathrm{DBP}$ \\
\hline Basu [40] & 8 weeks & Parallel arms & Controlled & $\begin{array}{c}48 \text { with MetS, age } 50 \pm 3, \text { BMI } \\
38 \pm 2\end{array}$ & $\begin{array}{c}\text { Freeze-dried blueberry } \\
\text { beverage (control: water) }\end{array}$ & $742 \mathrm{mg}$ & $<\mathrm{SBP},<\mathrm{DBP}$ \\
\hline Broncel [41] & 8 weeks & Pre-post & - & $\begin{array}{l}25 \text { with MetS, age 42-65, BMI } \\
31 \pm 3\end{array}$ & Aronia extract & $300 \mathrm{mg}$ & $<\mathrm{SBP},<\mathrm{DBP}$ \\
\hline Chai [42] & 12 weeks & Parallel arms & Controlled & $\begin{array}{l}34 \text { overweight older adults, } \\
\text { age } 70 \pm 4, \text { BMI } 28 \pm 4\end{array}$ & $\begin{array}{c}\text { Tart cherry juice (control: } \\
\text { energy and sugar matched } \\
\text { drink) }\end{array}$ & $\begin{array}{l}\text { (451 mg total } \\
\text { phenolics) }\end{array}$ & $<\mathrm{SBP},=\mathrm{DBP}$ \\
\hline Cook [43] & 12 days & Crossover & Controlled & $\begin{array}{c}15 \text { athletes, age } 38 \pm 12, \mathrm{BW} \\
76 \pm 10\end{array}$ & $\begin{array}{l}\text { Blackcurrant extract } \\
\text { (control: no extract) }\end{array}$ & $\begin{array}{l}105,210, \text { or } \\
315 \mathrm{mg}\end{array}$ & $\begin{array}{c}=\mathrm{SBP},=\mathrm{DBP},<\mathrm{MAP} \text { with } \\
210 \text { and } 315\end{array}$ \\
\hline Cook [44] & 1 week & Crossover & $\begin{array}{c}\text { Double-blind, } \\
\text { placebo-controlled }\end{array}$ & $\begin{array}{c}13 \text { healthy male, age } 26 \pm 4 \\
\text { BMI } 25 \pm 3\end{array}$ & Blackcurrant extract & $315 \mathrm{mg}$ & $\begin{array}{c}=\mathrm{SBP},=\mathrm{DBP},=\mathrm{MAP} \text { at rest }, \\
<\mathrm{SBP},<\mathrm{DBP},<\mathrm{MAP} \text { during } \\
\text { isomeric contraction }\end{array}$ \\
\hline Curtis [45] & 12 weeks & Parallel arms & $\begin{array}{l}\text { Double-blind, } \\
\text { placebo-controlled }\end{array}$ & $\begin{array}{c}52 \text { healthy postmenopausal } \\
\text { women, age } 58 \pm 6 \text {, BMI } 25 \pm \\
4\end{array}$ & $\begin{array}{l}\text { ACN-rich elderberry } \\
\text { extract capsule }\end{array}$ & $500 \mathrm{mg}$ & $=\mathrm{SBP},=\mathrm{DBP}$ \\
\hline Davinelli [46] & 4 weeks & Parallel arms & $\begin{array}{l}\text { Double-blind, } \\
\text { placebo-controlled }\end{array}$ & $\begin{array}{c}42 \text { overweight, age } 45-65 \\
\text { BMI } 29 \pm 4\end{array}$ & $\begin{array}{c}\text { ACN-rich maqui berry } \\
\text { extract }\end{array}$ & $486 \mathrm{mg}$ & $=\mathrm{SBP},=\mathrm{DBP}$ \\
\hline Desai [47] & 20 days & Parallel arms & $\begin{array}{l}\text { Single-blind, } \\
\text { placebo-controlled }\end{array}$ & $\begin{array}{c}11 \text { healthy, age } 30 \pm 10 \text {, BMI } \\
24 \pm 3\end{array}$ & Tart cherry juice & $540 \mathrm{mg}$ & $\begin{aligned}= & \mathrm{SBP},= \\
& =\text { DBP pre or post } \\
& \text { exercise }\end{aligned}$ \\
\hline Draijer [48] & 4 weeks & Crossover & $\begin{array}{l}\text { Double-blind, } \\
\text { placebo-controlled }\end{array}$ & $\begin{array}{l}60 \text { mildly hypertensive, age } \\
58 \pm 10 \text {, BMI } 26 \pm 4\end{array}$ & $\begin{array}{l}\text { Grape and grape wine } \\
\text { extracts } \\
\text { Berry mix (bilberries }\end{array}$ & $\begin{array}{l}\text { (800 mg total } \\
\text { phenolics) }\end{array}$ & $\begin{array}{l}<\mathrm{SBP},<\mathrm{DBP} \text { with grape } \\
\text { wine but not grape alone }\end{array}$ \\
\hline Erlund [49] & 8 weeks & Parallel arms & $\begin{array}{l}\text { Single-blind, } \\
\text { placebo-controlled }\end{array}$ & $\begin{array}{c}72 \text { with CVD risk factors, age } \\
58 \pm 6, \text { BMI } 26 \pm 3\end{array}$ & $\begin{array}{c}\text { lingonberries, black } \\
\text { currant, strawberry, } \\
\text { chokeberry, and raspberry) }\end{array}$ & $515 \mathrm{mg}$ & $<\mathrm{SBP},=\mathrm{DBP}$ \\
\hline Gurrola-Diaz [50] & 4 weeks & Parallel arms & Controlled & $\begin{array}{l}73 \text { healthy and } 51 \text { MetS } \\
\text { patients, age } 49 \pm 7, \mathrm{BMI} \\
29 \pm 5\end{array}$ & $\begin{array}{l}\text { Hibiscus sabdariffa extract } \\
\text { powder (control: } \\
\text { preventive diet) }\end{array}$ & $19 \mathrm{mg}$ & $\begin{array}{c}=\text { SBP },=\text { DBP in healthy and } \\
\text { MetS patients }\end{array}$ \\
\hline Habanova [51] & 6 weeks & Pre-post & - & $\begin{array}{c}36 \text { healthy, age } 48 \pm 6 \text {, BMI } \\
27 \pm 4\end{array}$ & $\begin{array}{c}\text { Frozen bilberries, } 3 \text { times a } \\
\text { week }\end{array}$ & $\begin{array}{l}456 \mathrm{mg}, 3 \\
\text { times/week }\end{array}$ & $=\mathrm{SBP},=\mathrm{DBP}$ \\
\hline Hassellund [52] & 4 weeks & Crossover & $\begin{array}{l}\text { Double-blind, } \\
\text { placebo-controlled }\end{array}$ & $\begin{array}{l}31 \text { moderately hypertensive } \\
\text { male, age } 41 \pm 3 \text {, BMI } 27 \pm 3\end{array}$ & $\begin{array}{l}\text { ACN capsule (isolated from } \\
\text { bilberry and blackcurrant) }\end{array}$ & $640 \mathrm{mg}$ & $\begin{array}{c}=\text { sitting, supine, or } \\
24 \mathrm{~h}-\text { ambulatory blood } \\
\text { pressure, or blood pressure } \\
\text { during stress test }\end{array}$ \\
\hline Jeong [53] & 8 weeks & Parallel arms & $\begin{array}{c}\text { Double-blind, } \\
\text { placebo-controlled }\end{array}$ & $\begin{array}{c}45 \text { prehypertensive, age } \\
57 \pm 12 \text {, BMI } 25 \pm 3\end{array}$ & $\begin{array}{l}\text { Dried unripe black } \\
\text { raspberry powder }\end{array}$ & $\begin{array}{l}\text { (1500 mg or } \\
2500 \mathrm{mg} \\
\text { powder) }\end{array}$ & $\begin{array}{c}<\text { SBP with high dose, } \\
=\text { DBP }\end{array}$ \\
\hline
\end{tabular}


Table 2. Cont

\begin{tabular}{|c|c|c|c|c|c|c|c|}
\hline Reference & Duration & Study Design & Control & Participants $^{\text {a }}$ & Source of ACN & $\begin{array}{l}\text { Daily Dose } \\
\text { of ACN }{ }^{b}\end{array}$ & Effect on Blood Pressure $^{c}$ \\
\hline Jeong [54] & 12 weeks & Parallel arms & $\begin{array}{l}\text { Double-blind, } \\
\text { placebo-controlled }\end{array}$ & $\begin{array}{c}51 \text { with MetS, age } 59 \pm 10 \\
\text { BMI } 25 \pm 4 \\
48 \text { postmenopausal women }\end{array}$ & $\begin{array}{l}\text { Dried unripe black } \\
\text { raspberry powder }\end{array}$ & $\begin{array}{l}\text { (750 } \mathrm{mg} \text { of } \\
\text { dry powder) }\end{array}$ & $=\mathrm{SBP},=\mathrm{DBP}$ \\
\hline Johnson [55] & 8 weeks & Parallel arms & $\begin{array}{l}\text { Double-blind, } \\
\text { placebo-controlled }\end{array}$ & $\begin{array}{c}\text { with pre- and stage } \\
\text { 1-hypertension, age } 59 \pm 5 \\
\text { BMI } 31 \pm 6\end{array}$ & $\begin{array}{l}\text { Freeze-dried blueberry } \\
\text { powder mixed with water }\end{array}$ & $469 \mathrm{mg}$ & $<\mathrm{SBP},<\mathrm{DBP}$ \\
\hline Kardum [56] & 4 weeks & Pre-post & - & $\begin{array}{c}20 \text { abdominally obese } \\
\text { postmenopausal women, age } \\
53 \pm 5, \text { BMI } 36 \pm 4\end{array}$ & $\begin{array}{l}\text { Glucomannan-enriched } \\
\text { aronia juice-based } \\
\text { supplement }\end{array}$ & $25 \mathrm{mg}$ & $<\mathrm{SBP},=\mathrm{DBP}$ \\
\hline Kardum [57] & 12 weeks & Pre-post & - & $\begin{array}{l}29 \text { healthy women, age } 35 \pm 8, \\
\text { BMI } 23 \pm 4\end{array}$ & $\begin{array}{l}\text { Glucomannan-enriched } \\
\text { aronia juice-based } \\
\text { supplement }\end{array}$ & $25 \mathrm{mg}$ & $=\mathrm{SBP},=\mathrm{DBP}$ \\
\hline Kardum [58] & 4 weeks & Pre-post & - & $\begin{array}{c}23 \text { pre- or stage } 1 \\
\text { hypertensive, age } 48 \pm 10 \\
\text { weight } 82 \pm 20\end{array}$ & Aronia juice & $358 \mathrm{mg}$ & $\begin{array}{c}<\mathrm{SBP},<\mathrm{DBP},<\text { average } 24 \mathrm{~h} \\
\mathrm{BP}\end{array}$ \\
\hline Karlsen [59] & 3 weeks & Parallel arms & Placebo-controlled & $\begin{array}{c}118 \text { adults, age } 61 \pm ?, \text { BMI } 25 \\
\pm ?\end{array}$ & $\begin{array}{c}\text { Purified ACN capsule from } \\
\text { bilberry and blackcurrant }\end{array}$ & 300 mg & $=\mathrm{SBP},=\mathrm{DBP}$ \\
\hline Kelley [60] & 4 weeks & Pre-post & - & $\begin{array}{c}18 \text { healthy, age } 50 \pm 4 \text {, BMI } 26 \\
\pm 4\end{array}$ & Fresh sweet cherries & $\begin{array}{l}\text { (280 g fresh } \\
\text { cherries) }\end{array}$ & $\begin{array}{l}=\mathrm{SBP},=\mathrm{DBP} \text { at the end of } \\
\text { the trial and after } 1 \text { month }\end{array}$ \\
\hline Kent [61] & 12 weeks & Parallel arms & Controlled & $\begin{array}{c}49 \text { older adults, age } 80 \pm 6 \\
\text { BMI } 26 \pm 3\end{array}$ & $\begin{array}{l}\text { Cherry juice (control: ACN } \\
\text { free apple juice) }\end{array}$ & $138 \mathrm{mg}$ & $<\mathrm{SBP},=\mathrm{DBP}$ \\
\hline Khan [62] & 6 weeks & Parallel arms & Placebo-controlled & $\begin{array}{c}66 \text { healthy adults, age } 52 \pm 10, \\
\text { BMI } 29 \pm 6\end{array}$ & $\begin{array}{c}\text { Blackcurrant juice, low or } \\
\text { high dose }\end{array}$ & $\begin{array}{c}40 \mathrm{mg} \text { or } 143 \\
\mathrm{mg}\end{array}$ & $=\mathrm{SBP},=\mathrm{DBP}$ \\
\hline Kolehmanen [63] & 8 weeks & Parallel arms & Controlled & $\begin{array}{l}27 \text { with MetS, age } 51 \pm 6 \text {, BMI } \\
32 \pm 4\end{array}$ & Bilberries (400 $\mathrm{g}$ fresh) & $1381 \mathrm{mg}$ & $=\mathrm{SBP},=\mathrm{DBP}$ \\
\hline Lehtonen [64] & 20 weeks & Parallel arms & Controlled & $\begin{array}{c}61 \text { women, age } 43 \pm \text { ?, BMI } 29 \\
\pm ?\end{array}$ & $\begin{array}{l}163 \mathrm{~g} \text { mix of } 18 \text { berries } \\
\text { (control: lifestyle } \\
\text { intervention) }\end{array}$ & $\begin{array}{l}\text { (equivalent } \\
\text { to } 163 \mathrm{~g} \\
\text { fresh berries) }\end{array}$ & $=\mathrm{SBP},=\mathrm{DBP}$ \\
\hline Lee [65] & 8 weeks & Parallel arms & $\begin{array}{c}\text { Double-blind, } \\
\text { placebo-controlled }\end{array}$ & $\begin{array}{c}63 \text { overweight or obese, age } \\
31 \pm 9, \text { BMI } 28 \pm 2\end{array}$ & $\begin{array}{c}\text { ACN-rich black soybean } \\
\text { extract }\end{array}$ & $31.45 \mathrm{mg}$ & $=\mathrm{SBP},=\mathrm{DBP}$ \\
\hline $\operatorname{Li}[66]$ & 24 weeks & Parallel arms & $\begin{array}{l}\text { Double-blind, } \\
\text { placebo-controlled }\end{array}$ & $\begin{array}{l}58 \text { with type II diabetes, age } \\
58 \pm 3, \text { BMI } 24 \pm 3\end{array}$ & $\begin{array}{c}\text { ACN capsules (isolated } \\
\text { from bilberry and black } \\
\text { currant) }\end{array}$ & $320 \mathrm{mg}$ & $<\mathrm{SBP},=\mathrm{DBP}$ \\
\hline Loo [67] & 16 weeks & Crossover & $\begin{array}{l}\text { Single-blind, } \\
\text { placebo-controlled }\end{array}$ & $\begin{array}{l}37 \text { with mild hypertension, } \\
\text { age } 40-70, \text { BMI } 26 \pm 3\end{array}$ & $\begin{array}{l}\text { Cold-pressed Aronia juice } \\
\text { and oven-dried Aronia } \\
\text { powder }\end{array}$ & $1024 \mathrm{mg}$ & $\begin{array}{l}<\text { daytime DBP (recorded } \\
\text { over } 15 \text { hours), =SBP }\end{array}$ \\
\hline Lynn [68] & 6 weeks & Parallel arms & Controlled & $\begin{array}{c}47 \text { healthy adults, age } 38 \pm 6 \\
\text { BMI } 24 \pm 3\end{array}$ & $\begin{array}{c}\text { Tart cherry concentrate, } \\
\text { (control: energy matched } \\
\text { drink) }\end{array}$ & $274.5 \mathrm{mg}$ & $=\mathrm{SBP},=\mathrm{DBP}$ \\
\hline Matsumoto [35] & 2 weeks & Crossover & $\begin{array}{l}\text { Double-blind, } \\
\text { placebo-controlled }\end{array}$ & 11 healthy, age $39 \pm 12$, BMI? & Blackcurrant ACN extract & $(7.7 \mathrm{mg} / \mathrm{kg})$ & $\begin{array}{c}=\mathrm{SBP},=\mathrm{DBP} \text { after } 30 \\
\text { minutes typing workload }\end{array}$ \\
\hline McAnulty [69] & 3 weeks & Parallel arms & Controlled & $\begin{array}{c}20 \text { smokers, age } 28 \pm 4, \text { BMI } \\
29 \pm 3\end{array}$ & Blueberry $250 \mathrm{~g}$ & & $=\mathrm{SBP},=\mathrm{DBP},=\mathrm{ACE}$ activity \\
\hline
\end{tabular}


Table 2. Cont.

\begin{tabular}{|c|c|c|c|c|c|c|c|}
\hline Reference & Duration & Study Design & Control & Participants $^{\text {a }}$ & Source of ACN & $\begin{array}{l}\text { Daily Dose } \\
\text { of ACN }\end{array}$ & Effect on Blood Pressure $^{c}$ \\
\hline McAnulty [70] & 6 weeks & Parallel arms & Placebo-controlled & $\begin{array}{l}25 \text { healthy, age } 43 \pm 12 \text {, BMI } \\
26 \pm 4\end{array}$ & $\begin{array}{c}\text { Freeze-dried blueberry } \\
\text { powder, equivalent to } 250 \mathrm{~g} \\
\text { berries }\end{array}$ & & $\begin{array}{c}<\text { aortic systolic pressures, } \\
<\text { SBP, }=\text { DBP, }<\text { DBP in the } \\
\text { subset of prehypertensive } \\
\text { subjects (9 subjects) }\end{array}$ \\
\hline Naruszewicz [71] & 6 weeks & Parallel arms & $\begin{array}{l}\text { Double-blind, } \\
\text { placebo-controlled }\end{array}$ & $\begin{array}{l}44 \text { post myocardial infarction } \\
\text { patients, receiving statin } \\
\text { therapy, age } 66 \pm 8, \text { BMI } 27 \pm \\
3\end{array}$ & $\begin{array}{c}255 \mathrm{mg} / \text { day Aronia } \\
\text { flavonoid extract }\end{array}$ & $64 \mathrm{mg}$ & $<\mathrm{SBP},<\mathrm{DBP}$ \\
\hline Nilsson [72] & 5 weeks & Crossover & Controlled & $\begin{array}{c}40 \text { healthy, age } 63 \pm 1 \text {, BMI } 24 \\
\pm 1\end{array}$ & $\begin{array}{c}\text { Mixed berry drink (1/3 } \\
\text { blueberries, } 1 / 9 \\
\text { blackcurrant, } 1 / 9 \text { elderberry, } \\
\text { 1/9 lingonberry, } 1 / 9 \\
\text { strawberry, } 2 / 9 \text { tomato), or } \\
\text { sugar-matched control }\end{array}$ & $248 \mathrm{mg}$ & $=\mathrm{SBP},=\mathrm{DBP}$ \\
\hline Ohguro [73] & 4 weeks & Crossover & $\begin{array}{l}\text { Double-blind, } \\
\text { placebo-controlled }\end{array}$ & 12 healthy, age $39 \pm 8$, BMI? & Blackcurrant ACN extract & $50 \mathrm{mg}$ & $\begin{array}{c}<\text { intraocular pressure, } \\
=\mathrm{SBP},=\mathrm{DBP}\end{array}$ \\
\hline Ohguro [74] & 2 years & Parallel arms & $\begin{array}{l}\text { Double-blind, } \\
\text { placebo-controlled }\end{array}$ & $\begin{array}{c}21 \text { glaucoma patients, age } 61 \\
\pm 7, \mathrm{BMI} ?\end{array}$ & Blackcurrant ACN extract & $50 \mathrm{mg}$ & $\begin{array}{c}<\text { intraocular pressure, } \\
=\text { SBP, }=\text { DBP }\end{array}$ \\
\hline Qin [75] & 12 weeks & Parallel arms & $\begin{array}{l}\text { Double-blind, } \\
\text { placebo-controlled }\end{array}$ & $\begin{array}{c}120 \text { dyslipidemic, age } 55 \pm 5 \\
\text { BMI } 26 \pm 4\end{array}$ & $\begin{array}{c}\text { ACN capsule (from } \\
\text { bilberry and blackcurrant) }\end{array}$ & $160 \mathrm{mg}$ & $=\mathrm{SBP},=\mathrm{DBP}$ \\
\hline $\begin{array}{l}\text { Puupponen-Pimia } \\
{[76]}\end{array}$ & 8 weeks & Parallel arms & Controlled & $\begin{array}{c}37 \text { overweight with MetS, age } \\
51 \pm 7, \text { BMI } 32 \pm 4\end{array}$ & $\begin{array}{l}300 \mathrm{~g} \text { frozen berries } \\
\text { (raspberries, strawberries, } \\
\text { and cloudberries) }\end{array}$ & $\begin{array}{l}\text { (equivalent } \\
\text { to } 300 \mathrm{~g} \\
\text { fresh berries) }\end{array}$ & $=\mathrm{SBP},=\mathrm{DBP}$ \\
\hline Riso [77] & 6 weeks & Crossover & Placebo-controlled & $\begin{array}{l}18 \text { male with risk factors for } \\
\text { CVD, age } 48 \pm 10 \text {, BMI } 25 \pm 3\end{array}$ & Wild blueberry drink & $400 \mathrm{mg}$ & $=\mathrm{SBP},=\mathrm{DBP}$ \\
\hline Skoczynska [78] & 6 weeks & Pre-post & - & $\begin{array}{c}\text { hypercholesterolemia, age } 54 \\
\pm 6 \text {, BMI } 28 \pm 3\end{array}$ & Aronia juice $250 \mathrm{~mL}$ & $90 \mathrm{mg}$ & $\begin{array}{c}<\text { SBP after } 12 \text { weeks, }<\text { DBP } \\
\text { after } 6 \text { and } 12 \text { weeks }\end{array}$ \\
\hline Sikora [79] & 8 weeks & Pre-post & - & $\begin{array}{l}23 \text { with untreated MetS (BMI } \\
31 \pm 4 \text { ), reference group with } \\
25 \text { treated MetS patients (BMI } \\
29 \pm 3 \text { ) and } 20 \text { healthy } \\
\text { controls (BMI } 23 \pm 1 \text { ) }\end{array}$ & $\begin{array}{l}\text { Aronia extract supplements } \\
\text { or ACE-inhibitors }\end{array}$ & $60 \mathrm{mg}$ & $<\mathrm{SBP},<\mathrm{DBP},<\mathrm{ACE}$ activity \\
\hline Stull [80] & 6 weeks & Parallel arms & $\begin{array}{l}\text { Double-blind, } \\
\text { placebo-controlled }\end{array}$ & $\begin{array}{l}32 \text { obese with } \\
\text { insulin-resistance, age } 52 \pm 3 \\
\text { BMI } 37 \pm 1\end{array}$ & $\begin{array}{c}\text { Blueberry powder added to } \\
\text { smoothie smoothie and } \\
\text { yogurt }\end{array}$ & $580 \mathrm{mg}$ & $=\mathrm{SBP},=\mathrm{DBP}$ \\
\hline Stull [81] & 6 weeks & Parallel arms & $\begin{array}{c}\text { Double-blind, } \\
\text { placebo-controlled }\end{array}$ & $\begin{array}{c}44 \text { adults, age } 57 \pm 2, \text { BMI } \\
36 \pm 1\end{array}$ & $\begin{array}{l}\text { Blueberry powder added to } \\
\text { smoothie and yogurt }\end{array}$ & $580 \mathrm{mg}$ & $=\mathrm{SBP},=\mathrm{DBP}$ \\
\hline Thompson [82] & 4 weeks & Crossover & $\begin{array}{c}\text { Double-blind, } \\
\text { placebo-controlled }\end{array}$ & $\begin{array}{c}16 \text { sedentary, age } 38 \pm 12 \\
\text { BMI } 23 \pm 2\end{array}$ & ACN capsule & $320 \mathrm{mg}$ & $=\mathrm{SBP},=\mathrm{DBP}$ \\
\hline
\end{tabular}


Table 2. Cont.

\begin{tabular}{|c|c|c|c|c|c|c|c|}
\hline Reference & Duration & Study Design & Control & Participants $^{a}$ & Source of $\mathrm{ACN}$ & $\begin{array}{l}\text { Daily Dose } \\
\text { of ACN }^{\text {b }}\end{array}$ & Effect on Blood Pressure $^{c}$ \\
\hline Tjelle [83] & 12 weeks & Parallel arms & $\begin{array}{l}\text { Double-blind, } \\
\text { placebo-controlled }\end{array}$ & $\begin{array}{c}134 \text { prehypertensive or } \\
\text { hypertensive, age } 52 \pm 6 \\
\text { BMI } 26 \pm 3\end{array}$ & $\begin{array}{l}\text { Mixed berry fruit juice (red } \\
\text { grape, aronia, cherry, and } \\
\text { bilberry) or same juice } \\
\text { enriched with black currant } \\
\text { press residue }\end{array}$ & $\begin{array}{l}43 \mathrm{mg} \text { or } \\
210 \mathrm{mg}\end{array}$ & $\begin{array}{l}<\mathrm{SBP} \text { in both juice groups, } \\
\text { more pronounced if high } \\
\text { BP baseline value, =DBP }\end{array}$ \\
\hline Udani [84] & 4 weeks & Pre-post & - & $\begin{array}{c}10 \text { overweight, age } 28 \pm ? \\
\text { BMI } 27 \pm 2\end{array}$ & $100 \mathrm{~g}$ açai pulp & $\begin{array}{l}0.77 \mathrm{mg} / \mathrm{mL} \\
\mathrm{ACN} \\
\text { (density } \\
\text { unknown) }\end{array}$ & $=\mathrm{SBP},=\mathrm{DBP}$ \\
\hline Whyte [85] & 6 months & Parallel arms & $\begin{array}{l}\text { Double-blind, } \\
\text { placebo-controlled }\end{array}$ & $\begin{array}{c}122 \text { older adults, age } 71 \pm 4 \\
\text { BW } 71 \pm 4\end{array}$ & $\begin{array}{l}\text { Whole wild blueberry } \\
\text { powder } 1 \text { or } 2 \mathrm{~g} \text {, or extract } \\
200 \mathrm{mg}\end{array}$ & $\begin{array}{l}2.7, \text { or } 5.4 \text { or } \\
\quad 14 \mathrm{mg}\end{array}$ & $\begin{array}{c}<\text { SBP with extract, but not } \\
\text { with powders, at } 3 \text { and } \\
6 \text { months }\end{array}$ \\
\hline Xie [86] & 12 weeks & Parallel arms & Placebo-controlled & $\begin{array}{l}49 \text { healthy former smokers, } \\
\text { age } 35 \pm 3, \text { BMI } 26 \pm 1\end{array}$ & 500 mg aronia extract & $45.1 \mathrm{mg}$ & $=\mathrm{SBP},=\mathrm{DBP}$ \\
\hline Zhang [87] & 12 weeks & Parallel arms & $\begin{array}{l}\text { Double-blind, } \\
\text { placebo-controlled }\end{array}$ & $\begin{array}{l}72 \text { patients with nonalcoholic } \\
\text { fatty liver disease, age } 46 \pm 8, \\
\text { BMI } 27 \pm 3\end{array}$ & $\begin{array}{l}\text { ACN capsules (isolated } \\
\text { from bilberry and } \\
\text { black currant) }\end{array}$ & $320 \mathrm{mg}$ & $=\mathrm{SBP},=\mathrm{DBP}$ \\
\hline Zhu [88] & 24 weeks & Parallel arms & $\begin{array}{l}\text { Double-blind, } \\
\text { placebo-controlled }\end{array}$ & $\begin{array}{l}146 \text { hypercholesterolemic, } \\
\text { age } 56 \pm 6 \text {, BMI } 27 \pm 2\end{array}$ & $\begin{array}{l}\text { ACN capsules (isolated } \\
\text { from bilberry and } \\
\text { black currant) }\end{array}$ & $320 \mathrm{mg}$ & $=\mathrm{SBP},=\mathrm{DBP}$ \\
\hline
\end{tabular}

${ }^{a}$ Age (in years) and BMI (in $\mathrm{kg} / \mathrm{m}^{2}$ ) data expressed as mean \pm SD. When data was reported as SEM, SD was calculated as SEM*SQRTparticipant. When BMI was unreported, weight in kg is reported instead. A question mark (?) indicates unreported data. ${ }^{b}$ When daily ACN dose is unreported, the best alternative information is reported in parentheses. ${ }^{\mathrm{c}}$ Only blood pressure data is reported in this table. Other outcomes of the studies are not reported. SBP: systolic blood pressure; DPB: diastolic blood pressure; MetS: Metabolic Syndrome; MAP: mean arterial pressure; ACE: angiotensin-converting enzyme; > or <: statistically significant increase or decrease; =: no significant change. 


\section{Potential Factors Influencing ACN Effects on Blood Pressure}

Several factors appear to influence the effects of ACNs on blood pressure regulation in humans, and they are discussed in the following subsections.

\subsection{Baseline Characteristics of the Population}

A consistent observation is that the effect on blood pressure is only detectable in subjects with high blood pressure baseline value. Of the six studies targeting specifically prehypertensive or hypertensive subjects, five found an effect on blood pressure [48,53,58,67,83], and only one did not [52]. In contrast, of the 16 studies enrolling completely healthy subjects with normal blood pressure, only one [68] found an effect on blood pressure, while the remaining 15 studies did not find any effect (Table 2).

After a six-week intervention with high bush blueberries on 25 adults, McAnulty et al. did not find a significant effect on DBP for the whole group, but the reduction became significant when considering only the subset of nine prehypertensive subjects [70]. Similarly, after an eight-week intervention with a mix of berries providing $515 \mathrm{mg}$ ACNs, on 72 subjects with CVD risk factors, Erlund et al. found an overall significant reduction in SBP, but observed that the effect was very strong in the subset of hypertensive participants [49]. Following 12 weeks consumption of a mixed berry fruit juice, a group of 134 prehypertensive or hypertensive subjects had a significant reduction in SBP, but the reduction was more pronounced in the subset of participants with higher baseline blood pressure values [83].

Another interesting observation comes from the study of Cook et al. on 13 healthy participants receiving a blackcurrant extract providing $315 \mathrm{mg}$ ACNs for a week [44]. While no effect on blood pressure was observed at rest, a significant reduction in SBP, DBP, and MAP was observed during isometric contraction, suggesting once more that ACNs act to lower BP when it is higher than normal, but do not lower it when it is already in a healthy range [44].

Based on these findings, future studies should aim to identify with more precision which population segments may benefit from $\mathrm{ACN}$ intake, possibly relating the findings to the new American Heart Association guidelines' blood pressure categories of normal $(<120 /<80 \mathrm{mmHg})$, elevated (120-129/<80 mmHg), stage 1 hypertension (130-139/80-89), stage 2 hypertension ( $\geq 140 / \geq 90 \mathrm{mmHg}$ ), and hypertensive crisis (>180/>120 $\mathrm{mmHg}$ ).

Another potentially relevant factor is the baseline total flavonoid intake of the population under study. It is reasonable to hypothesize that the effect of ACN supplementation may be greater in subjects with low baseline flavonoid intake. Unfortunately, this information is rarely collected or reported in studies, making it difficult to identify a pattern.

\subsection{Dose Effect}

Of the seven studies providing high doses of $\mathrm{ACN}(>500 \mathrm{mg} /$ day), four studies found a significant effect on blood pressure $[38,40,49,67]$, while the other three studies did not find any significant effect $[63,80,81]$. In contrast, a significant blood pressure-lowering effect was detected by three studies providing low doses of $\mathrm{ACN}(<100 \mathrm{mg} /$ day) $[56,71,79]$.

It is important to consider, however, that $\mathrm{ACN}$ quantification is rather complicated, and only few studies rely on complete ACN profiles of the food under investigation. More frequently, studies measure cyanidin-3-glycoside equivalents, or rely on a quick but approximate spectrophotometric quantification following methanol extraction. In some cases, they only estimate $\mathrm{ACN}$ content based on the USDA food composition tables. To further complicate matters, ACNs are rather unstable compounds and sensitive to $\mathrm{pH}$ variations, heat, light, oxygen exposure, and enzyme activity, and they are highly reactive with other molecules, sugars, proteins, and other phenolics [4]. Thus, the food matrix, its chemical composition, the manufacturing process, storage conditions, and duration, may all significantly affect $\mathrm{ACN}$ content and activity at the time of consumption even when the initial quantification is accurate [89-91]. Indeed, when Rodriguez-Mateos et al. tested both a freeze-dried blueberry drink and a blueberry baked product prepared with the same amount of blueberry powder, 
the ACN content of the drink was $339 \mathrm{mg}$, while only $196 \mathrm{mg} \mathrm{ACN}$ was found in the baked blueberry product; the rest being converted to other phenolics [37]. Thus, the reported ACN content from different studies may very easily be under- or overestimated, making it extremely difficult to compare studies.

Only seven studies investigated specifically the effect of different ACN doses, of which four seem to suggest the existence of a dose-effect. After 12 days consumption of a blackcurrant extract providing either 105, 210, or $315 \mathrm{mg} /$ day of ACNs, with a crossover design, a significant reduction in mean arterial pressure in a group of 15 athletes was only observed with the two higher ACN doses [43]. After an 8-week intervention with 1.5 or $2.5 \mathrm{~g}$ a day of black raspberry powder given to 45 prehypertensive subjects, a significant reduction of SBP was only observed in the group receiving the higher raspberry dose [53]. In a 6-week intervention with 122 older adults, Whyte et al. investigated the effects of either 1 or 2 daily grams of whole wild blueberry powder, or a $200 \mathrm{mg}$ wild blueberry extract, providing 2.7, 5.4, or $14 \mathrm{mg}$ ACNs, respectively. A significant reduction of SBP was only observed with the extract providing the higher ACN dose, but not with the whole berry powder [85].

In contrast, in another 6-week intervention with 66 mostly overweight adults, neither a low nor a higher dose blackcurrant juice (providing either $40 \mathrm{mg}$ or $143 \mathrm{mg}$ ACN daily, respectively) had any effect on blood pressure [62]. An intervention of 12 weeks on 134 prehypertensive or hypertensive subjects tested a mixed berry fruit juice providing $43 \mathrm{mg}$ ACNs, or the same juice enriched with blackcurrant press residues providing $210 \mathrm{mg}$ ACNs, and both juices significantly lowered SBP with no dose-dependency effect [83]. When 23 healthy participants received a single dose black-currant extract drink containing 150, 300, or $600 \mathrm{mg}$ ACNs following a high-carbohydrate meal, no significant changes in blood pressure were observed for any of the doses, two hours after the challenge [27].

Another interesting observation comes from the study of Kent et al. providing a single dose of cherry juice containing $207 \mathrm{mg}$ ACNs to a group of young or older adults and detecting a significant reduction of both SBP and DBP at two hours after consumption. However, when the same amount of juice was split into three doses provided one hour apart over two hours, the effect on blood pressure was no longer detectable [34].

Thus, a positive effect on blood pressure was found across a wide range of ACN doses, with both dose and time being relevant factors.

\subsection{Study Duration}

The study duration does not seem a consistent factor in determining the effect of ACNs on blood pressure. Of the 34 studies with 6 week durations or longer, 15 found a significant effect on blood pressure (Table 2). Of the 19 studies with durations of 4 weeks or less, seven studies found an effect (Table 2). Thus, a blood pressure-lowering effect is found both in longer and shorter studies. Indeed, a significant effect on blood pressure can be found also following single-dose interventions (Table 1).

It is interesting to note, however, that after 6-week consumption of aronia juice providing $90 \mathrm{mg} /$ day ACNs, a group of 58 male with mild hypercholesterolemia had a significant reduction in DBP but not in SBP, while after another 6 weeks of aronia juice consumption the reduction became significant also in SBP, suggesting that the duration of the intervention may indeed be a relevant factor [78].

\subsection{Systolic vs. Diastolic Blood Pressure}

Of the 28 studies registering a significant effect of blood pressure, 14 studies reported a reduction in SBP but not in DBP (Table 2), and 12 studies reported a reduction in both SBP and DBP (Table 2). Only one study found an effect on DBP but not in SBP, following a 16 weeks consumption of cold-pressed aronia juice and oven-dried aronia powder, providing $1024 \mathrm{mg} /$ day of ACNs, to 37 subjects with mild hypertension [67].

Thus, the blood-pressure-lowering effect appears to be more evident on SBP than DBP. 


\subsection{Effect on Angiotensin-Converting Enzyme (ACE)}

Hormonal long-term regulation of blood pressure, mainly via the renin-angiotensin-aldosterone and antidiuretic hormone (ADH) systems, is a potential target of ACN activity, as has been suggested by investigations in the animal model [92]. In humans, two studies have measured the effects of ACN consumption on the ACE.

A group of 23 subjects with untreated metabolic syndrome (MetS) received either aronia extract supplements providing $60 \mathrm{mg}$ ACNs, or ACE-inhibitors. After 8 weeks, the activity of SBP, DBP, and ACE was significantly lower compared to baseline. However, ACE activity was still higher compared to a reference group of healthy controls or MetS controls treated with ACE-inhibitors (reference group was measured only once and did not undertake any intervention) [79]. Conversely, following 3-week consumption of $250 \mathrm{~g}$ fresh blueberries, a group of 20 overweight smokers experienced no effect on blood pressure or ACE activity [36].

More studies on how ACN intake may affect blood pressure hormonal regulatory systems are warranted, and the variability in biological responses should also be considered in view of the recent findings on the M235T polymorphism of the angiotensinogen gene, which has been linked with cardiovascular disease [93].

\subsection{Synergistic Effects}

Many studies which found significant effects on BP used ACN-rich foods or whole extracts, which also contain other bioactive phytochemicals potentially affecting blood pressure. The exact composition of such extract is often unknown in most studies. Of the 14 studies using isolated ACNs or ACN-rich extracts, only one found a significant effect on blood pressure, while the remaining 13 studies did not find any effect. In contrast, of the 11 studies using whole phenolic extracts, eight studies found a significant effect, while only three studies did not find any effect on blood pressure (see Table 2).

It is also interesting to report that while no effect on blood pressure was observed when whole grapes were given for 4 weeks to a group of 60 mildly hypertensive participants, a significant reduction of both SBP and DBP was detected with grape wine extract that had a comparable total phenolic content [48]. Thus, berry phenolics-but not isolated ACNs-appear to be effective on blood pressure, suggesting that the effect is synergistic with other molecules. Of course, it is also possible that the effect is entirely exerted by other phenolic compounds, or other phytonutrients, independent of ACNs, although as far as we know ACNs are the only molecules which are abundant and transversally present in all the different berries examined in this review, and for which positive effects on BP have been detected.

Furthermore, it is also to be noted that most of the studies testing isolated ACNs used the same commercially available supplement, made with ACNs isolated from bilberry and blackcurrant.

A synergistic effect with lifestyle and diet is also a likely relevant factor. Gurrola Diaz et al. tested the effect of 4-week consumption of a Hibiscus sabdariffa (HS) extract powder providing $19 \mathrm{mg}$ ACN on a group of 71 healthy and 51 MetS patients, using a preventive diet as control. No significant effect on blood pressure was found either with HS alone or with diet alone. However, in the group receiving both the HS powder and the preventive diet, a significant reduction of both SBP and DBP was observed [50].

\subsection{Differential Effect of Individual ACNs}

Most interventions use fresh berries or berry extracts, which contain a mix of different compounds including different ACNs. This is undoubtedly the best approach in view of extrapolating the results to a real-life situation, in which whole foods are consumed and not isolated compounds, and the consumption of whole foods rather than supplements should be encouraged to promote health and prevent disease. However, this approach makes it more difficult to identify potential differential effects among different molecules, especially when trying to elucidate their mechanisms of action. 
Indeed, when studying single anthocyanins, Rechner \& Kroner observed that the inhibitory effect on the redox-sensitive p38 MAPK and c-jun-N-terminal kinase pathways often reported for ACNs, was only caused by delphinidin and cyanidin, but not malvidin and peonidin, suggesting that the hydroxyl residue in position 3 of the B ring may play a key functional role [94]. Thus, it is not unreasonable to hypothesize that the variability in the results between different studies is also at least in part due to the fact that some ACNs have a stronger effect on BP than others.

The different $\mathrm{ACN}$ profile of individual food sources is also a factor of variability. For example, blueberries contain predominantly delphinidin, malvidin, and petunidin; raspberries predominantly cyanidin and pelargonidin; and blackberries predominantly cyanidin and malvidin [14]. It must be noted, however, that significant effects on blood pressure have been observed with all different berries, including chokeberries, blueberries, raspberries, cherries, and blackcurrants (Tables 1 and 2).

\subsection{ACN Absorption and Metabolism}

ACN metabolism is complex and mostly unknown, and in order to fully understand their biological functions, it is necessary to better elucidate their metabolic fate [90]. While most in vitro studies focus on isolated ACNs, it is important to remember that only less than $1 \%$ of total dietary ACNs are absorbed intact. A higher proportion of dietary ACNs is absorbed after hydrolysis and partial degradation to other phenolic compounds. Part of the unabsorbed ACNs is also fermented by the colonic microbiota, and their catabolic products are subsequently absorbed into the bloodstream [95]. Furthermore, ACNs are quite unstable at neutral $\mathrm{pH}$, and after they are absorbed, $\mathrm{ACN}$ parent compounds, degradation products and microbial metabolites all undergo significant metabolism by both phase I and phase II enzymes to form methyl, glucuronide, and sulfate conjugated metabolites [12].

Thus, it is not enough to study the biological effects of parent ACNs, but also the effects of their numerous catabolic products based on microbiota, physiology, and health of the GI in individuals. This likely explains most of the discrepancies between the mechanisms of action suggested in vitro and the actual in vivo outcomes.

\subsection{Interaction with Gut Microbiota}

Increasing evidence links the composition of gut microbiota to key physiological effects related to the prevention of chronic disease, and this includes the contribution of gut bacteria to blood pressure regulation $[96,97]$. The relationship between ACNs and gut bacteria goes both ways: on one hand, ACN intake influences the composition of gut microbiome, and on the other hand, colonic fermentations transform unabsorbed ACNs to different catabolic products that can be absorbed and act as bioactives [98]. Microbial catabolism of ACNs consists mainly in the cleavage of their heterocyclic flavylium ring (the C-ring), and subsequent dehydroxylation or decarboxylation to form phenolic acids $[97,99]$.

Thus, interaction with gut microbiome is likely an important element of variability that could explain some of the different effects observed for ACN intake. To our knowledge, however, no study has directly investigated the relationship between gut microbiota and the effects of ACN intake on blood pressure regulation.

\section{Conclusions}

A consistent number of studies documented a significant blood-pressure-lowering activity related to ACNs and ACN-rich berry consumption, suggesting that an effect indeed exists.

The fact that many other studies failed to observe such an effect, indicates that the outcome is not generalized and likely depends on many other factors, and, in particular, the baseline characteristics of the population (more specifically, their baseline blood pressure and total flavonoid intake), ACN dose, duration of the intervention, differential effects of individual ACNs, and synergistic effects with other phenolics and bioactive phytochemicals in general. Additionally, ACN content and bioavailability from the food matrix (whole food, juice, freeze-dried powder, and extract), modified by the manufacturing 
process and storage conditions and duration, need to be taken into account. Finally, ACN absorption and metabolism, which is affected by the different microbiota enterotypes of each individual, his/her different genotype, the physiological condition of the gastrointestinal tract and its relative response to dietary bioactives, is also a factor to be considered.

Further research will need to identify more precisely the clinical conditions and the characteristics of individuals for which an increased consumption of $\mathrm{ACN}$-rich foods may be especially recommended and could potentially reduce the dose and/or the administration of antihypertensive medications.

Author Contributions: Both authors conceived and planned the outline of the review. S.V. conducted the literature search and drafted the manuscript. D.K.-Z. reviewed and completed the manuscript.

Funding: This research received no external funding.

Conflicts of Interest: The authors declare no conflicts of interest.

\section{References}

1. Clark, J.L.; Zahradka, P.; Taylor, C.G. Efficacy of flavonoids in the management of high blood pressure. Nutr. Rev. 2015, 73, 799-822. [CrossRef] [PubMed]

2. Cicero, A.F.G.; Fogacci, F.; Colletti, A. Food and plant bioactives for reducing cardiometabolic disease risk: An evidence based approach. Food Funct. 2017, 8, 2076-2088. [CrossRef] [PubMed]

3. Cicero, A.F.G.; Grassi, D.; Tocci, G.; Galletti, F.; Borghi, C.; Ferri, C. Nutrients and Nutraceuticals for the Management of High Normal Blood Pressure: An Evidence-Based Consensus Document. High Blood Press Cardiovasc. Prev. 2019, 26, 9-25. [CrossRef] [PubMed]

4. De Ferrars, R.M.; Czank, C.; Zhang, Q.; Botting, N.P.; Kroon, P.A.; Cassidy, A.; Kay, C.D. The pharmacokinetics of anthocyanins and their metabolites in humans. Br. J. Pharmacol. 2014, 171, 3268-3282. [CrossRef] [PubMed]

5. Stoner, G.D.; Sardo, C.; Apseloff, G.; Mullet, D.; Wargo, W.; Pound, V.; Singh, A.; Sanders, J.; Aziz, R.; Casto, B.; et al. Pharmacokinetics of anthocyanins and ellagic acid in healthy volunteers fed freeze-dried black raspberries daily for 7 days. J. Clin. Pharmacol. 2005, 45, 1153-1164. [CrossRef] [PubMed]

6. Wu, X.; Beecher, G.R.; Holden, J.M.; Haytowitz, D.B.; Gebhardt, S.E.; Prior, R.L. Concentrations of anthocyanins in common foods in the United States and estimation of normal consumption. J. Agric. Food Chem. 2006, 54, 4069-4075. [CrossRef] [PubMed]

7. Wallace, T.C. Anthocyanins in cardiovascular disease. Adv. Nutr. 2011, 2, 1-7. [CrossRef]

8. Mink, P.J.; Scrafford, C.G.; Barraj, L.M.; Harnack, L.; Hong, C.P.; Nettleton, J.A.; Jacobs, D.R., Jr. Flavonoid intake and cardiovascular disease mortality: A prospective study in postmenopausal women. Am. J. Clin. Nutr. 2007, 85, 895-909. [CrossRef]

9. Vendrame, S.; Klimis-Zacas, D. Anti-inflammatory effect of anthocyanins via modulation of nuclear factor- $\mathrm{kB}$ and mitogen-activated protein kinase signaling cascades. Nutr. Rev. 2015, 73, 348-358. [CrossRef]

10. Vendrame, S.; Del Bo', C.; Ciappellano, S.; Riso, P.; Klimis-Zacas, D. Berry Fruit Consumption and Metabolic Syndrome. Antioxidants 2016, 5, 34. [CrossRef]

11. Jurikova, T.; Mlcek, J.; Skrovankova, S.; Sumczynski, D.; Sochor, J.; Hlavacova, I.; Snopek, L.; Orsavova, J. Fruits of Black Chokeberry Aronia melanocarpa in the Prevention of Chronic Diseases. Molecules 2017, $22,944$. [CrossRef] [PubMed]

12. García-Conesa, M.T.; Chambers, K.; Combet, E.; Pinto, P.; Garcia-Aloy, M.; Andrés-Lacueva, C.; de Pascual-Teresa, S.; Mena, P.; Konic Ristic, A.; Hollands, W.J.; et al. Meta-Analysis of the Effects of Foods and Derived Products Containing Ellagitannins and Anthocyanins on Cardiometabolic Biomarkers: Analysis of Factors Influencing Variability of the Individual Responses. Int. J. Mol. Sci. 2018, $19,694$. [CrossRef]

13. Kelley, D.S.; Adkins, Y.; Laugero, K.D. A Review of the Health Benefits of Cherries. Nutrients 2018, 10, 368. [CrossRef]

14. Corona, G.; Tang, F.; Vauzour, D.; Rodriguez-Mateos, A.; Spencer, J. Assessment of the anthocyanidin content of common fruits and development of a test diet rich in a range of anthocyanins. J. Berry Res. 2011, 1, $209-216$. [CrossRef]

15. Bell, D.R.; Gochenaur, K. Direct vasoactive and vasoprotective properties of anthocyanin-rich extracts. J. Appl. Physiol. 2006, 100, 1164-1170. [CrossRef] 
16. Kim, J.N.; Han, S.N.; Ha, T.J.; Kim, H.K. Black soybean anthocyanins attenuate inflammatory responses by suppressing reactive oxygen species production and mitogen activated protein kinases signaling in lipopolysaccharide-stimulated macrophages. Nutr. Res. Pract. 2017, 11, 357-364. [CrossRef] [PubMed]

17. Parichatikanond, W.; Pinthong, D.; Mangmool, S. Blockade of the renin-angiotensin system with delphinidin, cyanin, and quercetin. Planta Med. 2012, 78, 1626-1632. [CrossRef]

18. Qin, Y.; Zhai, Q.; Li, Y.; Cao, M.; Xu, Y.; Zhao, K.; Wang, T. Cyanidin-3-O-glucoside ameliorates diabetic nephropathy through regulation of glutathione pool. Biomed. Pharmacother. 2018, 103, 1223-1230. [CrossRef] [PubMed]

19. Cassidy, A.; O’Reilly, É.J.; Kay, C.; Sampson, L.; Franz, M.; Forman, J.P.; Curhan, G.; Rimm, E.B. Habitual intake of flavonoid subclasses and incident hypertension in adults. Am. J. Clin. Nutr. 2011, 93, 338-347. [CrossRef] [PubMed]

20. Jennings, A.; Welch, A.A.; Fairweather-Tait, S.J.; Kay, C.; Minihane, A.M.; Chowienczyk, P.; Jiang, B.; Cecelja, M.; Spector, T.; Macgregor, A.; et al. Higher anthocyanin intake is associated with lower arterial stiffness and central blood pressure in women. Am. J. Clin. Nutr. 2012, 96, 781-788. [CrossRef] [PubMed]

21. Huang, H.; Chen, G.; Liao, D.; Zhu, Y.; Xue, X. Effects of Berries Consumption on Cardiovascular Risk Factors: A Meta-analysis with Trial Sequential Analysis of Randomized Controlled Trials. Sci. Rep. 2016, 6, 23625. [CrossRef]

22. Yang, L.; Ling, W.; Du, Z.; Chen, Y.; Li, D.; Deng, S.; Liu, Z.; Yang, L. Effects of Anthocyanins on Cardiometabolic Health: A Systematic Review and Meta-Analysis of Randomized Controlled Trials. Adv. Nutr. 2017, 8, 684-693. [CrossRef] [PubMed]

23. Daneshzad, E.; Shab-Bidar, S.; Mohammadpour, Z.; Djafarian, K. Effect of anthocyanin supplementation on cardio-metabolic biomarkers: A systematic review and meta-analysis of randomized controlled trials. Clin. Nutr. 2019, 38, 1153-1165. [CrossRef] [PubMed]

24. Zhu, Y.; Bo, Y.; Wang, X.; Lu, W.; Wang, X.; Han, Z.; Qiu, C. The Effect of Anthocyanins on Blood Pressure: A PRISMA-Compliant Meta-Analysis of Randomized Clinical Trials. Medicine (Baltimore) 2016, 95, e3380. [CrossRef] [PubMed]

25. Zhu, Y.; Sun, J.; Lu, W.; Wang, X.; Wang, X.; Han, Z.; Qiu, C. Effects of blueberry supplementation on blood pressure: A systematic review and meta-analysis of randomized clinical trials. J. Hum. Hypertens. 2017, 31, 165-171. [CrossRef]

26. Alqurashi, R.M.; Galante, L.A.; Rowland, I.R.; Spencer, J.P.; Commane, D.M. Consumption of a flavonoid-rich açai meal is associated with acute improvements in vascular function and a reduction in total oxidative status in healthy overweight men. Am. J. Clin. Nutr. 2016, 104, 1227-1235. [CrossRef]

27. Castro-Acosta, M.L.; Smith, L.; Miller, R.J.; McCarthy, D.I.; Farrimond, J.A.; Hall, W.L. Drinks containing anthocyanin-rich blackcurrant extract decrease postprandial blood glucose, insulin and incretin concentrations. J. Nutr. Biochem. 2016, 38, 154-161. [CrossRef]

28. Del Bo', C.; Porrini, M.; Fracassetti, D.; Campolo, J.; Klimis-Zacas, D.; Riso, P. A single serving of blueberry (V. corymbosum) modulates peripheral arterial dysfunction induced by acute cigarette smoking in young volunteers: A randomized-controlled trial. Food Funct. 2014, 5, 3107-3116. [CrossRef]

29. Del Bo', C.; Deon, V.; Campolo, J.; Lanti, C.; Parolini, M.; Porrini, M.; Klimis-Zacas, D.; Riso, P. A serving of blueberry ( $V$. corymbosum) acutely improves peripheral arterial dysfunction in young smokers and non-smokers: Two randomized, controlled, crossover pilot studies. Food Funct. 2017, 8, 4108-4117. [CrossRef]

30. Igwe, E.O.; Charlton, K.E.; Roodenrys, S.; Kent, K.; Fanning, K.; Netzel, M.E. Anthocyanin-rich plum juice reduces ambulatory blood pressure but not acute cognitive function in younger and older adults: A pilot crossover dose-timing study. Nutr. Res. 2017, 47, 28-43. [CrossRef]

31. Keane, K.M.; George, T.W.; Constantinou, C.L.; Brown, M.A.; Clifford, T.; Howatson, G. Effects of Montmorency tart cherry (Prunus Cerasus, L.) consumption on vascular function in men with early hypertension. Am. J. Clin. Nutr. 2016, 103, 1531-1539. [CrossRef] [PubMed]

32. Keane, K.M.; Haskell-Ramsay, C.F.; Veasey, R.C.; Howatson, G. Montmorency Tart cherries (Prunus cerasus L.) modulate vascular function acutely, in the absence of improvement in cognitive performance. Br. J. Nutr. 2016, 116, 1935-1944. [CrossRef] [PubMed] 
33. Keane, K.M.; Bailey, S.J.; Vanhatalo, A.; Jones, A.M.; Howatson, G. Effects of montmorency tart cherry (L. Prunus Cerasus) consumption on nitric oxide biomarkers and exercise performance. Scand. J. Med. Sci. Sports 2018, 28, 1746-1756. [CrossRef] [PubMed]

34. Kent, K.; Charlton, K.E.; Jenner, A.; Roodenrys, S. Acute reduction in blood pressure following consumption of anthocyanin-rich cherry juice may be dose-interval dependant: A pilot cross-over study. Int. J. Food Sci. Nutr. 2016, 67, 47-52. [CrossRef] [PubMed]

35. Matsumoto, H.; Takenami, E.; Iwasaki-Kurashige, K.; Osada, T.; Katsumura, T.; Hamaoka, T. Effects of blackcurrant anthocyanin intake on peripheral muscle circulation during typing work in humans. Eur. J. Appl. Physiol. 2005, 94, 36-45. [CrossRef] [PubMed]

36. Rodriguez-Mateos, A.; Rendeiro, C.; Bergillos-Meca, T.; Tabatabaee, S.; George, T.W.; Heiss, C.; Spencer, J.P. Intake and time dependence of blueberry flavonoid-induced improvements in vascular function: A randomized, controlled, double-blind, crossover intervention study with mechanistic insights into biological activity. Am. J. Clin. Nutr. 2013, 98, 1179-1191. [CrossRef] [PubMed]

37. Rodriguez-Mateos, A.; Del Pino-García, R.; George, T.W.; Vidal-Diez, A.; Heiss, C.; Spencer, J.P. Impact of processing on the bioavailability and vascular effects of blueberry (poly)phenols. Mol. Nutr. Food Res. 2014, 58, 1952-1961. [CrossRef] [PubMed]

38. Ataie-Jafari, A.; Hosseini, S.; Karimi, F.; Pajouhi, M. Effects of sour cherry juice on blood glucose and some cardiovascular risk factors improvements in diabetic women: A pilot study. Nutr. Food Sci. 2008, 38, 355-360. [CrossRef]

39. Barona, J.; Aristizabal, J.C.; Blesso, C.N.; Volek, J.S.; Fernandez, M.L. Grape polyphenols reduce blood pressure and increase flow-mediated vasodilation in men with metabolic syndrome. J. Nutr. 2012, 142, 1626-1632. [CrossRef]

40. Basu, A.; Du, M.; Leyva, M.J.; Sanchez, K.; Betts, N.M.; Wu, M.; Aston, C.E.; Lyons, T.J. Blueberries decrease cardiovascular risk factors in obese men and women with metabolic syndrome. J. Nutr. 2010, 140, 1582-1587. [CrossRef]

41. Broncel, M.; Kozirog, M.; Duchnowicz, P.; Koter-Michalak, M.; Sikora, J.; Chojnowska-Jezierska, J. Aronia melanocarpa extract reduces blood pressure, serum endothelin, lipid, and oxidative stress marker levels in patients with metabolic syndrome. Med. Sci. Monit. 2010, 16, CR28-CR34. [PubMed]

42. Chai, S.C.; Davis, K.; Wright, R.S.; Kuczmarski, M.F.; Zhang, Z. Impact of tart cherry juice on systolic blood pressure and low-density lipoprotein cholesterol in older adults: A randomized controlled trial. Food Funct. 2018, 9, 3185-3194. [CrossRef]

43. Cook, M.D.; Myers, S.D.; Gault, M.L.; Edwards, V.C.; Willems, M.E. Cardiovascular function during supine rest in endurance-trained males with New Zealand blackcurrant: A dose-response study. Eur. J. Appl. Physiol. 2017, 117, 247-254. [CrossRef] [PubMed]

44. Cook, M.D.; Myers, S.D.; Gault, M.L.; Willems, M.E.T. Blackcurrant Alters Physiological Responses and Femoral Artery Diameter during Sustained Isometric Contraction. Nutrients 2017, 9, 556. [CrossRef]

45. Curtis, P.J.; Kroon, P.A.; Hollands, W.J.; Walls, R.; Jenkins, G.; Kay, C.D.; Cassidy, A. Cardiovascular disease risk biomarkers and liver and kidney function are not altered in postmenopausal women after ingesting an elderberry extract rich in anthocyanins for 12 weeks. J. Nutr. 2009, 139, 2266-2271. [CrossRef] [PubMed]

46. Davinelli, S.; Bertoglio, J.C.; Zarrelli, A.; Pina, R.; Scapagnini, G. A Randomized Clinical Trial Evaluating the Efficacy of an Anthocyanin-Maqui Berry Extract (Delphinol@) on Oxidative Stress Biomarkers. J. Am. Coll. Nutr. 2015, 34, 28-33. [CrossRef] [PubMed]

47. Desai, T.; Bottoms, L.; Roberts, M. The effects of Montmorency tart cherry juice supplementation and FATMAX exercise on fat oxidation rates and cardio-metabolic markers in healthy humans. Eur. J. Appl. Physiol. 2018, 118, 2523-2539. [CrossRef]

48. Draijer, R.; de Graaf, Y.; Slettenaar, M.; de Groot, E.; Wright, C.I. Consumption of a polyphenol-rich grape-wine extract lowers ambulatory blood pressure in mildly hypertensive subjects. Nutrients 2015, 7, 3138-3153. [CrossRef]

49. Erlund, I.; Koli, R.; Alfthan, G.; Marniemi, J.; Puukka, P.; Mustonen, P.; Mattila, P.; Jula, A. Favorable effects of berry consumption on platelet function; blood pressure; and HDL cholesterol. Am. J. Clin. Nutr. 2008, 87, 323-331. [CrossRef] 
50. Gurrola-Díaz, C.M.; García-López, P.M.; Sánchez-Enríquez, S.; Troyo-Sanromán, R.; Andrade-González, I.; Gómez-Leyva, J.F. Effects of Hibiscus sabdariffa extract powder and preventive treatment (diet) on the lipid profiles of patients with metabolic syndrome (MeSy). Phytomedicine 2010, 17, 500-505. [CrossRef]

51. Habanova, M.; Saraiva, J.A.; Haban, M.; Schwarzova, M.; Chlebo, P.; Predna, L.; Gažo, J.; Wyka, J. Intake of bilberries (Vaccinium myrtillus L.) reduced risk factors for cardiovascular disease by inducing favorable changes in lipoprotein profiles. Nutr. Res. 2016, 36, 1415-1422. [CrossRef] [PubMed]

52. Hassellund, S.S.; Flaa, A.; Sandvik, L.; Kjeldsen, S.E.; Rostrup, M. Effects of anthocyanins on blood pressure and stress reactivity: A double-blind randomized placebo-controlled crossover study. J. Hum. Hypertens. 2012, 26, 396-404. [CrossRef] [PubMed]

53. Jeong, H.S.; Kim, S.; Hong, S.J.; Choi, S.C.; Choi, J.H.; Kim, J.H.; Park, C.Y.; Cho, J.Y.; Lee, T.B.; Kwon, J.W.; et al. Black Raspberry Extract Increased Circulating Endothelial Progenitor Cells and Improved Arterial Stiffness in Patients with Metabolic Syndrome: A Randomized Controlled Trial. J. Med. Food 2016, 19, 346-352. [CrossRef]

54. Jeong, H.S.; Hong, S.J.; Cho, J.Y.; Lee, T.B.; Kwon, J.W.; Joo, H.J.; Park, J.H.; Yu, C.W.; Lim, D.S. Effects of Rubus occidentalis extract on blood pressure in patients with prehypertension: Randomized, double-blinded, placebo-controlled clinical trial. Nutrition 2016, 32, 461-467. [CrossRef] [PubMed]

55. Johnson, S.A.; Figueroa, A.; Navaei, N.; Wong, A.; Kalfon, R.; Ormsbee, L.T.; Feresin, R.G.; Elam, M.L.; Hooshmand, S.; Payton, M.E.; et al. Daily blueberry consumption improves blood pressure and arterial stiffness in postmenopausal women with pre- and stage 1-hypertension: A randomized, double-blind, placebo-controlled clinical trial. J. Acad. Nutr. Diet. 2015, 115, 369-377. [CrossRef]

56. Kardum, N.; Petrović-Oggiano, G.; Takic, M.; Glibetić, N.; Zec, M.; Debeljak-Martacic, J.; Konić-Ristić, A. Effects of glucomannan-enriched, aronia juice-based supplement on cellular antioxidant enzymes and membrane lipid status in subjects with abdominal obesity. Sci. World J. 2014, 2014, 869250. [CrossRef] [PubMed]

57. Kardum, N.; Konić-Ristić, A.; Savikin, K.; Spasić, S.; Stefanović, A.; Ivanišević, J.; Miljković, M. Effects of polyphenol-rich chokeberry juice on antioxidant/pro-oxidant status in healthy subjects. J. Med. Food 2014, 17, 869-874. [CrossRef]

58. Kardum, N.; Milovanović, B.; Šavikin, K.; Zdunić, G.; Mutavdžin, S.; Gligorijević, T.; Spasić, S. Beneficial Effects of Polyphenol-Rich Chokeberry Juice Consumption on Blood Pressure Level and Lipid Status in Hypertensive Subjects. J. Med. Food 2015, 18, 1231-1238. [CrossRef]

59. Karlsen, A.; Retterstøl, L.; Laake, P.; Paur, I.; Bøhn, S.K.; Sandvik, L.; Blomhoff, R. Anthocyanins inhibit nuclear factor-kappaB activation in monocytes and reduce plasma concentrations of pro-inflammatory mediators in healthy adults. J. Nutr. 2007, 137, 1951-1954. [CrossRef]

60. Kelley, D.S.; Adkins, Y.; Reddy, A.; Woodhouse, L.R.; Mackey, B.E.; Erickson, K.L. Sweet bing cherries lower circulating concentrations of markers for chronic inflammatory diseases in healthy humans. J. Nutr. 2013, 143, 340-344. [CrossRef]

61. Kent, K.; Charlton, K.; Roodenrys, S.; Batterham, M.; Potter, J.; Traynor, V.; Gilbert, H.; Morgan, O.; Richards, R. Consumption of anthocyanin-rich cherry juice for 12 weeks improves memory and cognition in older adults with mild-to-moderate dementia. Eur. J. Nutr. 2017, 56, 333-341. [CrossRef] [PubMed]

62. Khan, F.; Ray, S.; Craigie, A.M.; Kennedy, G.; Hill, A.; Barton, K.L.; Broughton, J.; Belch, J.J. Lowering of oxidative stress improves endothelial function in healthy subjects with habitually low intake of fruit and vegetables: A randomized controlled trial of antioxidant- and polyphenol-rich blackcurrant juice. Free Radic. Biol. Med. 2014, 72, 232-237. [CrossRef] [PubMed]

63. Kolehmainen, M.; Mykkänen, O.; Kirjavainen, P.V.; Leppänen, T.; Moilanen, E.; Adriaens, M.; Laaksonen, D.E.; Hallikainen, M.; Puupponen-Pimiä, R.; Pulkkinen, L.; et al. Bilberries reduce low-grade inflammation in individuals with features of metabolic syndrome. Mol. Nutr. Food Res. 2012, 56, 1501-1510. [CrossRef] [PubMed]

64. Lehtonen, H.M.; Suomela, J.P.; Tahvonen, R.; Yang, B.; Venojärvi, M.; Viikari, J.; Kallio, H. Different berries and berry fractions have various but slightly positive effects on the associated variables of metabolic diseases on overweight and obese women. Eur. J. Clin. Nutr. 2011, 65, 394-401. [CrossRef] [PubMed]

65. Lee, M.; Sorn, S.R.; Park, Y.; Park, H.K. Anthocyanin Rich-Black Soybean Testa Improved Visceral Fat and Plasma Lipid Profiles in Overweight/Obese Korean Adults: A Randomized Controlled Trial. J. Med. Food 2016, 19, 995-1003. [CrossRef] 
66. Li, D.; Zhang, Y.; Liu, Y.; Sun, R.; Xia, M. Purified anthocyanin supplementation reduces dyslipidemia; enhances antioxidant capacity; and prevents insulin resistance in diabetic patients. J. Nutr. 2015, 145, 742-748. [CrossRef] [PubMed]

67. Loo, B.M.; Erlund, I.; Koli, R.; Puukka, P.; Hellström, J.; Wähälä, K.; Mattila, P.; Jula, A. Consumption of chokeberry (Aronia mitschurinii) products modestly lowered blood pressure and reduced low-grade inflammation in patients with mildly elevated blood pressure. Nutr. Res. 2016, 36, 1222-1230. [CrossRef]

68. Lynn, A.; Mathew, S.; Moore, C.T.; Russell, J.; Robinson, E.; Soumpasi, V.; Barker, M.E. Effect of a tart cherry juice supplement on arterial stiffness and inflammation in healthy adults: A randomised controlled trial. Plant Foods Hum. Nutr. 2014, 69, 122-127. [CrossRef]

69. McAnulty, S.R.; McAnulty, L.S.; Morrow, J.D.; Khardouni, D.; Shooter, L.; Monk, J.; Gross, S.; Brown, V. Effect of daily fruit ingestion on angiotensin converting enzyme activity, blood pressure, and oxidative stress in chronic smokers. Free Radic. Res. 2005, 39, 1241-1248. [CrossRef]

70. McAnulty, L.S.; Collier, S.R.; Landram, M.J.; Whittaker, D.S.; Isaacs, S.E.; Klemka, J.M.; Cheek, S.L.; Arms, J.C.; McAnulty, S.R. Six weeks daily ingestion of whole blueberry powder increases natural killer cell counts and reduces arterial stiffness in sedentary males and females. Nutr. Res. 2014, 34, 577-584. [CrossRef]

71. Naruszewicz, M.; Laniewska, I.; Millo, B.; Dłuzniewski, M. Combination therapy of statin with flavonoids rich extract from chokeberry fruits enhanced reduction in cardiovascular risk markers in patients after myocardial infraction (MI). Atherosclerosis 2007, 194, e179-e184. [CrossRef] [PubMed]

72. Nilsson, A.; Salo, I.; Plaza, M.; Björck, I. Effects of a mixed berry beverage on cognitive functions and cardiometabolic risk markers; A randomized cross-over study in healthy older adults. PLoS ONE 2017, 12, e0188173. [CrossRef] [PubMed]

73. Ohguro, H.; Ohguro, I.; Yagi, S. Effects of black currant anthocyanins on intraocular pressure in healthy volunteers and patients with glaucoma. J. Ocul. Pharmacol. Ther. 2013, 29, 61-67. [CrossRef] [PubMed]

74. Ohguro, H.; Ohguro, I.; Katai, M.; Tanaka, S. Two-year randomized, placebo-controlled study of black currant anthocyanins on visual field in glaucoma. Ophthalmologica 2012, 228, 26-35. [CrossRef] [PubMed]

75. Qin, Y.; Xia, M.; Ma, J.; Hao, Y.; Liu, J.; Mou, H.; Cao, L.; Ling, W. Anthocyanin supplementation improves serum LDL- and HDL-cholesterol concentrations associated with the inhibition of cholesteryl ester transfer protein in dyslipidemic subjects. Am. J. Clin. Nutr. 2009, 90, 485-492. [CrossRef] [PubMed]

76. Puupponen-Pimiä, R.; Seppänen-Laakso, T.; Kankainen, M.; Maukonen, J.; Törrönen, R.; Kolehmainen, M.; Leppänen, T.; Moilanen, E.; Nohynek, L.; Aura, A.M.; et al. Effects of ellagitannin-rich berries on blood lipids; gut microbiota; and urolithin production in human subjects with symptoms of metabolic syndrome. Mol. Nutr. Food Res. 2013, 57, 2258-2263. [CrossRef]

77. Riso, P.; Klimis-Zacas, D.; Del Bo', C.; Martini, D.; Campolo, J.; Vendrame, S.; Møller, P.; Loft, S.; De Maria, R.; Porrini, M. Effect of a wild blueberry (Vaccinium angustifolium) drink intervention on markers of oxidative stress, inflammation and endothelial function in humans with cardiovascular risk factors. Eur. J. Nutr. 2013, 52, 949-961. [CrossRef]

78. Skoczynska, A.; Jedrychowska, I.; Poręba, R.; Affelska-Jercha, A.; Turczyn, B.; Wojakowska, A.; Andrzejak, R.; Jedrychowska-Bianchi, I. Influence of chokeberry juice on arterial blood pressure and lipid parameters in men with mild hypercholesterolemia. Pharmacol. Rep. 2007, 59, 177-182.

79. Sikora, J.; Broncel, M.; Mikiciuk-Olasik, E. Aronia melanocarpa Elliot reduces the activity of angiotensin i-converting enzyme-in vitro and ex vivo studies. Oxid. Med. Cell. Longev. 2014, 2014, 739721. [CrossRef]

80. Stull, A.J.; Cash, K.C.; Johnson, W.D.; Champagne, C.M.; Cefalu, W.T. Bioactives in Blueberries Improve Insulin Sensitivity in Obese; Insulin-Resistant Men and Women. J. Nutr. 2010, 140, 1764-1768. [CrossRef]

81. Stull, A.J.; Cash, K.C.; Champagne, C.M.; Gupta, A.K.; Boston, R.; Beyl, R.A.; Johnson, W.D.; Cefalu, W.T. Blueberries improve endothelial function, but not blood pressure, in adults with metabolic syndrome: A randomized, double-blind, placebo-controlled clinical trial. Nutrients 2015, 7, 4107-4123. [CrossRef]

82. Thompson, K.; Hosking, H.; Pederick, W.; Singh, I.; Santhakumar, A.B. The effect of anthocyanin supplementation in modulating platelet function in sedentary population: A randomised, double-blind, placebo-controlled, cross-over trial. Br. J. Nutr. 2017, 118, 368-374. [CrossRef]

83. Tjelle, T.E.; Holtung, L.; Bøhn, S.K.; Aaby, K.; Thoresen, M.; Wiik, S.Å.; Paur, I.; Karlsen, A.S.; Retterstøl, K.; Iversen, P.O.; et al. Polyphenol-rich juices reduce blood pressure measures in a randomised controlled trial in high normal and hypertensive volunteers. Br. J. Nutr. 2015, 114, 1054-1063. [CrossRef] 
84. Udani, J.K.; Singh, B.B.; Singh, V.; Barrett, M.L. Effects of Açai (Euterpe oleracea Mart.) berry preparation on metabolic parameters in a healthy overweight population: A pilot study. Nutr. J. 2011, 10, 45. [CrossRef]

85. Whyte, A.R.; Cheng, N.; Fromentin, E.; Williams, C.M. A Randomized, Double-Blinded, Placebo-Controlled Study to Compare the Safety and Efficacy of Low Dose Enhanced Wild Blueberry Powder and Wild Blueberry Extract (ThinkBlue ${ }^{\mathrm{TM}}$ ) in Maintenance of Episodic and Working Memory in Older Adults. Nutrients 2018, 10, 660. [CrossRef]

86. Xie, L.; Vance, T.; Kim, B.; Lee, S.G.; Caceres, C.; Wang, Y.; Hubert, P.A.; Lee, J.Y.; Chun, O.K.; Bolling, B.W. Aronia berry polyphenol consumption reduces plasma total and low-density lipoprotein cholesterol in former smokers without lowering biomarkers of inflammation and oxidative stress: A randomized controlled trial. Nutr. Res. 2017, 37, 67-77. [CrossRef]

87. Zhang, P.W.; Chen, F.X.; Li, D.; Ling, W.H.; Guo, H.H. A CONSORT-compliant; randomized; double-blind; placebo-controlled pilot trial of purified anthocyanin in patients with nonalcoholic fatty liver disease. Medicine (Baltimore) 2015, 94, e758. [CrossRef]

88. Zhu, Y.; Ling, W.; Guo, H.; Song, F.; Ye, Q.; Zou, T.; Li, D.; Zhang, Y.; Li, G.; Xiao, Y.; et al. Anti-inflammatory effect of purified dietary anthocyanin in adults with hypercholesterolemia: A randomized controlled trial. Nutr. Metab. Cardiovasc. Dis. 2013, 23, 843-849. [CrossRef]

89. Kay, C.D.; Mazza, G.; Holub, B.J.; Wang, J. Anthocyanin metabolites in human urine and serum. Br. J. Nutr. 2004, 91, 933-942. [CrossRef]

90. Mazza, G.; Kay, C.D.; Cottrell, T.; Holub, B.J. Absorption of anthocyanins from blueberries and serum antioxidant status in human subjects. J. Agric. Food Chem. 2002, 50, 7731-7737. [CrossRef]

91. Fracassetti, D.; Del Bo', C.; Simonetti, P.; Gardana, C.; Klimis-Zacas, D.; Ciappellano, S. Effect of Time and storage temperature on anthocyanin decay and antioxidant activity in wild blueberry (Vaccinium angustifolium) powder. J. Agric. Food Chem. 2013, 61, 2999-3005. [CrossRef]

92. Wiseman, W.; Egan, J.M.; Slemmer, J.E.; Shaughnessy, K.S.; Ballem, K.; Gottschall-Pass, K.T.; Sweeney, M.I. Feeding blueberry diets inhibits angiotensin II-converting enzyme (ACE) activity in spontaneously hypertensive stroke-prone rats. Can. J. Physiol. Pharmacol. 2011, 89, 67-71. [CrossRef]

93. Zhai, C.; Cong, H.; Zhang, H.; Hou, K.; Zhang, Y.; Zhang, Y. M235T polymorphism in the angiotensinogen gene and cardiovascular disease: An updated meta-analysis of 39 case-control comparisons. Anatol. J. Cardiol. 2019, 21, 222-232. [CrossRef]

94. Rechner, A.R.; Kroner, C. Anthocyanins and colonic metabolites of dietary polyphenols inhibit platelet function. Thromb. Res. 2005, 116, 327-334. [CrossRef]

95. McGhie, T.K.; Walton, M.C. The bioavailability and absorption of anthocyanins: Towards a better understanding. Mol. Nutr. Food Res. 2007, 51, 702-713. [CrossRef]

96. Jama, H.; Beale, A.; Shihata, W.A.; Marques, F.Z. The effect of diet on hypertensive pathology: Is there a link via gut microbiota-driven immune-metabolism? Cardiovasc. Res. 2019, cvz091. [CrossRef]

97. Williamson, G.; Clifford, M.N. Colonic metabolites of berry polyphenols: The missing link to biological activity? Br. J. Nutr. 2010, 104, S48-S66. [CrossRef]

98. Krga, I.; Tamaian, R.; Mercier, S.; Boby, C.; Monfoulet, L.E.; Glibetic, M.; Morand, C.; Milenkovic, D. Anthocyanins and their gut metabolites attenuate monocyte adhesion and transendothelial migration through nutrigenomic mechanisms regulating endothelial cell permeability. Free Radic. Biol. Med. 2018, 124, 364-379. [CrossRef]

99. Zhang, X.; Sandhu, A.; Edirisinghe, I.; Burton-Freeman, B. An exploratory study of red raspberry (Rubus idaeus L.) (poly)phenols/metabolites in human biological samples. Food Funct. 2018, 9, 806-818. [CrossRef]

(C) 2019 by the authors. Licensee MDPI, Basel, Switzerland. This article is an open access article distributed under the terms and conditions of the Creative Commons Attribution (CC BY) license (http://creativecommons.org/licenses/by/4.0/). 